\title{
Analysis of Cylindrical Antennas-A Spectral Iteration Technique
}

\author{
S. A. BOKHARI AND N. BALAKRISHNAN
}

\begin{abstract}
The cylindrical antenna problem has been tackled using the spectral iteration technique. An iterative scheme is employed for improving on an initially assumed form of the current distribution. Use is made of the fast Fourier transform (FFT) algorithm, and the cumbersome process of matrix inversion is circumvented. Consequently, this method is capable of handling a larger number of anknown coefficients in the expansion of the current distribution. Furthermore, it provides a convenient means of testing for the satisfaction of the boundary conditions on the surface of the antenna. Convergence criteria for the iteration process have been established and the use of an acceleration procedure is illustrated. Different types of source models have been investigated, and the convergence of both local and nonlocal parameters is also discussed.
\end{abstract}

\section{INTRODUCTION}

$\mathbf{T}$ HE CYLINDRICAL antenna is perhaps one of the most extensively investigated forms of antennas. Comprehensive accounts of the analysis techniques employed have appeared in several books including [1] and [2]. Most of these methods employ either Pocklington's integral equation or Hallen's integral equation and solve for the unknown current distribution. These techniques, despite subtle variations between them, can often be conveniently explained by the unifying theory of the method of moments [3]. The method of moments, as is well-known, reduces the integral equation to a matrix equation. The solution then requires the inversion of this matrix, the order of which is dependent upon the number of unknown functions used in representing the current distribution. Various choices of basis and testing functions have been extensively investigated [1], [4]. However, it is also well-known that the demonstrated accuracy of the solutions in the calculation of some guantities like the input impedance, radiation pattern etc., is no guarantee that the overall solution can be accepted as being physically realistic or correct. As a matter of fact, various aspects of the calculated results may be obviously invalid, but without a negative impact on the overall usefulness of the result. An example of this is illustrated by the computed near fields on the surface of a cylindrical antenna [4].

The near fields around antennas are of significant interest while analyzing antennas near obstacles, corona discharge assessment, electromagnetic pulse (EMP) vulnerability assessment, etc. King and $\mathrm{Wu}_{\mathrm{u}}$ [5] have considered the problem of computing the near fields of cylindrical driven antennas. However, since they employ Hallen's integral equation, computation of the near fields is not so straightforward. Their method calls for the evaluation of a complicated expression involving sine and cosine integrals for the near fields parallel to the cylinder axis. Neff et al. [6], by employing a trigonometric series expansion for the current distribution in the solution of Hallen's integral equation have utilized the Helmholtz integral for vector and scalar potentials and the equation of continuity in deriving a

Manuscript received December 23, 1983; revised June 14, 1984.

The authors are with the Department of Aerospace Engineering, Indian Institute of Science, Bangalore 560012, India. simpler expression for the near fields of a thin centerfed cylindrical antenna. Their results for a half-wave dipole compare well with those of King and Wu [5]. Miller and Deadrick [4] have computed the near fields on the surface of perfectly conducting cylindrical antennas with several choices of subdomain type basis and testing functions in their moment method solutions. They attribute the disparities observed in the near fields, with different basis functions to certain peculiarities associated with the basis functions employed. Commonly used basis functions of the subdomain type lead to currents which may have discontinuities in amplitude, slope etc. at segment junctions each of which can produce a distinctive variation in the near fields at the points of discontinuity. Since Pocklington's integral equation involves a second derivative of the current distribution, Anders [7] has suggested the usage of basis functions with continuous second derivatives to obtain a smooth field distribution along the surface of the antenna. Mittra and Klein [8] have also concluded that the choice of basis functions cannot be arbitrary and that certain choices may yield erroneous results. Therefore, a method of solution which checks for the satisfaction of boundary conditions preferably without involving excessive computational effort is desirable. Ko and Mittra [9] have developed a new iterative scheme in the Fourier transform domain which has a provision for checking the satisfaction of boundary conditions. Matrix inversion is circumvented in this method, and use is made of the fast Fourier transform algorithm thereby resulting in a computationally efficient solution. Although this technique has been successfully applied to a variety of scattering problems [10]-[12] convergence of the iteration process has not been discussed in detail. Moreover, application of this technique to cylindrical antenna problems has not been reported so far. Sarkar et al. [13] have investigated various numerical methods for the solution of large systems of linear equations arising electromagnetic field problems. Their results tend to favor iterative methods as opposed to matrix inversion techniques, and they have particularly suggested usage of the conjugate gradient method. However, they have not considered the spectral iteration technique in their discussion.

Although several different types of basis and testing functions have been investigated, one possibility that appears to have been overlooked is that of employing subdomain type basis functions for the expansion of the current distribution and entire domain type basis functions for testing. The method employed in this paper, in some sense, fills this gap.

In this paper we employ a similar scheme [9] for solving the cylindrical antenna problem. In order to bring out the advantages of this technique over the conventional moment method solutions, a new explanation is given in light of the method of moments itself. The mathematical significance of a particular truncation operator [9] and the choice of an acceleration scheme [10], [11] are clearly brought out. Criterion for the convergence of this method have been derived. A number of examples with different types of source fields have been worked out to 
bring out the effectiveness of this method. Convergence of both local and nonlocal parameters has also been discussed.

\section{METHOD OF SOLUTION}

Consider a straight, center-fed, cylindrical wire of circular cross sectional radius $a$ and length $2 h$ symmetrically located about the origin of a rectangular coordinate system and oriented along the $z$-axis. For simplicity we assume the usual thin wire approximations.

Pocklington's integral equation [8] is of the form

$\left[\frac{d^{2}}{d z^{2}}+\beta^{2}\right] A_{z}(z)=-j \omega \epsilon E_{z i}(z), \quad z \in[-h, h]$

where $\beta$ and $\epsilon$ are the propagation constant and permittivity of the medium, $E_{z i}(z)$ is the tangential component of the incident electric field and the vector potential $A_{z}(z)$ is given by

$$
A_{z}(z)=\int_{-h}^{h} J\left(z^{\prime}\right) G\left(z-z^{\prime}\right) d z^{\prime}
$$

where $J\left(z^{\prime}\right)$ denotes the current distribution ( $z$-directed) and free space Green's function $G\left(z-z^{\prime}\right)$ is given by

$$
G\left(z-z^{\prime}\right)=\frac{\exp \left[-j \beta \sqrt{\left.\left(z-z^{\prime}\right)^{2}+a^{2}\right]}\right.}{4 \pi \sqrt{\left(z-z^{\prime}\right)^{2}+a^{2}}} .
$$

Since (1) is valid on the wire only, to enable Fourier transformation, a truncation operator $\theta$ is defined as in [9]. For the onedimensional case considered here, $\theta$ can be taken as a pulse of unit amplitude and the operation it performs as a multiplication, i.e.,

$$
\begin{aligned}
\theta(z) & =1, & & -h \leqslant z \leqslant h \\
& =0, & & \text { otherwise. }
\end{aligned}
$$

Similarly a complementary operator $\hat{\theta}$ is defined as

$$
\begin{aligned}
\hat{\theta}(z) & =0, & & -h \leqslant z \leqslant h \\
& =1, & & \text { otherwise. }
\end{aligned}
$$

Equation (1) can now be written as

$$
\begin{aligned}
& C\left(\frac{d^{2}}{d z^{2}}+\beta^{2}\right)[G(z) * J(z)] \\
& =-\theta(z) E_{z i}(z)+C \hat{\theta}(z)\left[\frac{d^{2}}{d z^{2}}+\beta^{2}\right][G(z) * \theta(z) J(z)] \\
& z \\
& z \in[-\infty, \infty]
\end{aligned}
$$

where the asterisk denotes the convolution operation, $C=$ $-j \beta / Z, Z$ the intrinsic impedance of free space, and use is made of the identity [9]

$$
\theta(z) J(z)=J(z)
$$

which is obvious since the current distribution vanishes outside the antenna. However, in the iterative process employed, in the solution, this identity is helpful in several ways as will be discussed later.

We shall employ the following form for the Fourier transform:

$$
\tilde{H}=\tilde{H}(f)=F[H(z)]=\int_{-\infty}^{\infty} H(z) \exp (-j 2 \pi f z) d z
$$

and for the inverse Fourier transform

$$
H=H(z)=F^{-1}[\tilde{H}(f)]=\int_{-\infty}^{\infty} \tilde{H}(f) \exp (j 2 \pi f z) d f .
$$

Fourier transforming (6) on both sides, an iterative form for the transform of the current distribution can be written as [9]

$$
\begin{aligned}
\tilde{J}^{(k+1)}= & (\tilde{D} \tilde{G})^{-1}\left[F\left(-\theta E_{z i}\right)+F\left\{F^{-1}\left(\tilde{D} \tilde{G} F\left(\theta J^{k}\right)\right)\right.\right. \\
& \left.\left.-\theta F^{-1}\left(\tilde{D} \tilde{G} F\left(\theta J^{k}\right)\right)\right\}\right]
\end{aligned}
$$

where

$$
\begin{aligned}
& \tilde{D}=C\left[\beta^{2}-(2 \pi f)^{2}\right] \\
& \tilde{G}=\frac{K_{0}\left[a \sqrt{(2 \pi f)^{2}-\beta^{2}}\right]}{2 \pi} \\
& k=0,1,2, \cdots .
\end{aligned}
$$

$K_{0}$ denotes the modified Bessel function of second kind and zeroth order and the superscripts $k$ indicate the iteration numbers.

The solution then begins with an initial estimate for the current distribution $J^{0}$, evaluation of $F\left(\theta J^{0}\right)$, and, using (10) an improved approximation for $J^{1}$ is obtained. For the next iteration $J^{1}$ is obtained from $F^{-1}\left(\tilde{J}^{1}\right)$ and the process can be continued. The iteration process is explained in detail in [9]. After completion of each iteration, a boundary condition check can be made by comparing $F_{z}^{k}=\theta F^{-1}\left[\tilde{D} \tilde{G} F\left(\theta J^{k}\right)\right]$, with the incident electric field $E_{z i}$. Note that the satisfaction of the boundary condition itself can be used as a stopping criterion for the iteration process. Alternatively, we can compare $\widetilde{J}^{(k+1)}$ and $F\left(\theta J^{k}\right)$ after each iteration and stop the iteration process after the difference $\left[\widetilde{J}^{(k+1)}-F\left(\theta J^{k}\right)\right]$ is less than a predetermined quantity.

\section{SOURCE MODELING}

In performing antenna calculations, it is vitally important to introduce the proper exciting field. Here we employ three different types of source models, the Fourier transforms of which can be expressed in a closed form.

\section{A. Delta Gap Feed}

For a voltage generator of $V$ volts located at $z=0$,

$$
E_{z i}(z)=V \delta(z)
$$

and

$$
F\left(\theta E_{z i}\right)=\tilde{E}_{z i}=V
$$

where $\delta(z)$ denotes the delta function.

\section{B. Pulse Feed}

In this case, the electric field of the source is assumed to be uniformly distributed over a small region about the feed point.

$$
\begin{aligned}
E_{z i}(z) & =V / 2 z_{1}, & & -z_{1} \leqslant z \leqslant z_{1} \text { and } z_{1}<h \\
& =0, & & \text { otherwise }
\end{aligned}
$$

and

$$
F\left(\theta E_{z i}\right)=\tilde{E}_{z i}=V \operatorname{sinc}\left(2 \pi f z_{1}\right) .
$$




\section{Magnetic Frill Excitation}

For a magnetic frill voltage generator of $V$ volts located at $z=0$ the source field is of the form [4],

$$
\begin{aligned}
E_{z i}(z)= & V\left[\exp \left(-j \beta R_{a}\right) / R_{a}\right. \\
& \left.-\exp \left(-j \beta R_{b}\right) / R_{b}\right] / 2 \operatorname{Ln}(b / a)
\end{aligned}
$$

where

$$
\begin{array}{ll}
R_{x} & =\sqrt{x^{2}+z^{2}} \\
a & \text { frill inner radius } \\
b & \text { frill outer radius. }
\end{array}
$$

As long as the wire length $2 h$ and the ratio $(b / a)$ are not too small, $E_{z i}(z)$ can be regarded as essentially support limited to the range $(-h, h)$ and

$$
\begin{aligned}
F\left(\theta E_{z i}\right) \cong & \tilde{E}_{z i} \\
\cong & V\left[K_{0}\left[a \sqrt{(2 \pi f)^{2}-\beta^{2}}\right]\right. \\
& \left.\left.-K_{0}\left[b \sqrt{(2 \pi f)^{2}-\beta^{2}}\right)\right]\right] / \operatorname{Ln}(b / a) .
\end{aligned}
$$

\section{CONVERGENCE CRITERIA}

We now examine the conditions under which the iteration process given by (10) is convergent. We shall employ matrix notations since convergence criteria for iterative solutions are well established for matrix methods. Since the fast Fourier transform (FFT) algorithm is employed, certain points regarding the discrete Fourier transform (DFT) need to be made.

We first choose an appropriate sampling interval $\Delta z$, sufficiently small so as to keep the aliasing of the current distribution to a minimium. Let $N_{p}=2 h / \Delta z$ and $N_{p}+1$ denote a set of equally spaced points defined on the antenna as shown in Fig. 1. We next choose ati appropriate integer $N$, such that $N>N_{p}+1$. For the sake of the FFT, $N$ should be highly composite, preferably a power of two. We set $N=2^{i}, i$ an integer.

Eqautions (8) and (9) can be expressed in a discrete form as [14]

$$
\begin{aligned}
\tilde{H}_{p}(m \Delta f)=\Delta z \sum_{n=-N / 2}^{N / 2-1} H_{p}(n \Delta z) \exp (-j 2 \pi m n / N) \\
H_{p}(m \Delta z)=\Delta f \sum_{n=-N / 2}^{N / 2-1} \tilde{H}_{p}(n \Delta f) \exp (j 2 \pi m n / N) \\
-N / 2 \leqslant m<N / 2
\end{aligned}
$$

where $\tilde{H}_{p}(m \Delta f)$ and $H_{p}(m \Delta z)$, denote periodic representation of $\tilde{H}(f)$ and $H(z)$ and

$$
\Delta f=\frac{1}{N \Delta z} \text {. }
$$

Equations (18) and (19) can also be expressed in a matrix form. Let $m$ denote the rows and $n$ the columns of a matrix. For uniformity in notations we let $m$ and $n$ take values from $-N / 2$ to $(N / 2)-1$. A subscript $c$ will be used to indicate a column matrix. Therefore,

$$
\begin{aligned}
{\left[\tilde{H}_{p}\right]_{c} } & =\Delta z[W]\left[H_{p}\right]_{c} \\
{\left[H_{p}\right]_{c} } & =\Delta f[\bar{W}]\left[\widetilde{H}_{p}\right]_{c}
\end{aligned}
$$

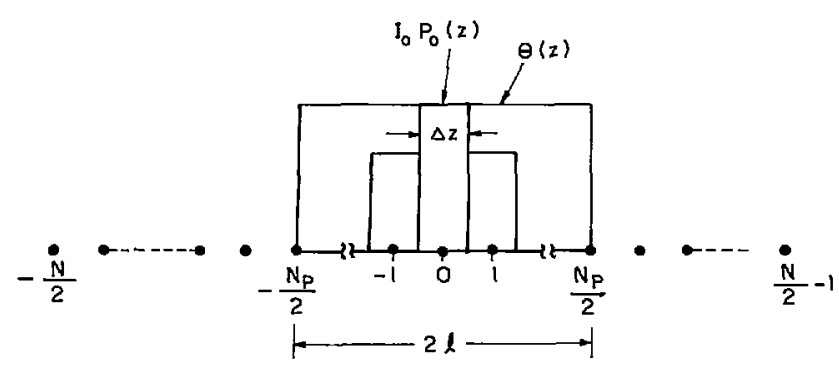

Fig. 1. Piecewise constant representation of the current distribution.

where the mnth elements of the corresponding matrices are

$$
\begin{gathered}
{\left[\tilde{H}_{p}\right]_{c}-\tilde{H}_{p}(m \Delta f)} \\
{\left[H_{p}\right]_{c}-H_{p}(m \Delta z)} \\
{[W]-\exp (-j 2 \pi m n / N)} \\
{[\overline{\mathscr{W}}]-\exp (j 2 \pi m n / N)}
\end{gathered}
$$

and

$$
\Delta z[W] \Delta f[\bar{W}]=[U]
$$

where $[U]$ denotes the identity matrix.

The basic equation to be solved is

$$
\begin{aligned}
& C \theta(z)\left[\frac{d^{2}}{d z^{2}}+\beta^{2}\right][G(z) *\{\theta(z) J(z)\}] \\
& =-\theta(z) E_{z i}(z) .
\end{aligned}
$$

Since using the DFT in the computation of the Fourier transform amounts to assuming a piecewise constant representation for both the function $H_{p}(m \Delta z)$ and the exponent in (18), we express $J$ in terms of piecewise constant basis functions. However, unlike moment method, $J$ is defined over the entire range $[-N / 2,(N / 2)-1]$ and the operator $\theta$ does the truncation over the antenna (Fig. 1).

$$
J=\sum_{n=-N / 2}^{N / 2-1} I_{n} P_{n}(z)
$$

where

$$
\begin{aligned}
P_{n}(z) & \dot{1} 1, & & n-\frac{\Delta z}{2} \leqslant z \leqslant n+\frac{\Delta z}{2} \\
& =0, & & \text { otherwise. }
\end{aligned}
$$

$P_{n}$ denote pulses of width $\Delta z$ and the $I_{n}$ are the constant coefficients to be determined. Note that the choice of pulses as basis functions does not meet the requirements of the current going to zero at the wire ends. This is usually overcome in moment method solutions by providing extra pulses at the wire ends with zero currents. We need not be concerned about this complexity here since when the iteration process to be employed in the solution converges, it gives a current distribution that is numerically zero at the wire ends. A delta function in the DFT sense can be treated as a pulse of width equal to the sampling interval $\Delta z$ and height $1 / \Delta z$. All $P_{n}$ can be related to $P_{0}$ as

$$
P_{n}(z)=\left[P_{\mathrm{o}}(z-n \Delta z) / \Delta z\right] \Delta z
$$

and

$$
\tilde{P}_{n}(m \Delta f)=\Delta z \exp (-j 2 \pi n \Delta z m \Delta f) .
$$


Using (25) and (26) in (24),

$$
\begin{aligned}
& C \Delta z \theta(z)\left[\frac{d^{2}}{d z^{2}}+\beta^{2}\right]\left[G(z) *\left\{\theta(z) \sum_{n=-N / 2}^{N / 2-1} I_{n} P_{0}(z-n \Delta z) / \Delta z\right\}\right] \\
& =-\theta(z) E_{z i}(z) .
\end{aligned}
$$

A set of weighting functions $\exp (-j 2 \pi m \Delta f z),-N / 2 \leqslant m<$ $N / 2$, are defined over the range $(-\infty, \infty)$. Taking the inner product on both sides of (27) with these weighting functions, (the equivalent of Fourier transforming) a matrix equation for the unknown currents can be written. Since the DFT is employed, the integration reduces to a summation and the range to $[-N / 2,(N / 2)-1]$. The weighting functions chosen can be regarded as of the entire domain type. However, as the term entire domain in moment method parlance usually refers to basis function defined over the entire domain of the antenna alone, here their domain is $(-\infty, \infty)$. Note that instead of matching the tangential electric field due to $J$, (say $F_{z}$ ) with $E_{z i}$ at certain specified points as in point matching or matching the averaged value of $F_{z}$ with a corresponding averaged $E_{z i}$ as in Galerkin's method, here, the spectrum of $F_{z}$ is matched with the spectrum of $E_{z i}$.

As mentioned in [9], a main advantage of dealing in the spectral domain is that the time consuming convolution integral in the spatial domain is reduced to an algebraic product of transforms in the spectral domain. In fact, this is an efficient algorithm for computing the convolution of two functions by taking the inverse DFT. of the product of their DFT's [15]. However, it must be remembered that due to the periodic representation of the function and its transform in the DFT, this operation results in circular convolution rather than the linear convolution that is desired. We shall for the time being assume that the convolution property of the continuous Fourier transform is valid for the DFT and we will later on show how circular convolution can be overcome by the truncation operator $\theta$.

Taking the Fourier transform on both sides of (27) and after some simplifications, the following matrix equation is obtained.

$$
[A][I]_{c}=[E]_{c} \text {. }
$$

where

$$
\begin{aligned}
{[A] } & =\left[A^{\prime}\right][\theta] \\
{\left[A^{\prime}\right] } & =C \Delta z[W][\theta] \Delta f[\bar{W}][D G] \Delta z[W]
\end{aligned}
$$

and the elements of the matrices are

$$
\begin{gathered}
{[\theta]-\theta(n \Delta z) \delta_{m}^{n}} \\
{[D G]-\tilde{D}(m \Delta f) \tilde{G}(m \Delta f) \delta_{m}^{n}} \\
{[I]_{c}-I_{m}} \\
{[E]_{c}--\theta \tilde{E}_{z i}(m \Delta f) .}
\end{gathered}
$$

$\delta_{m}^{n}$ denotes the Kronecker delta and is used to indicate a diagonal matrix.

Since we are interested in the current distribution on the antenna alone, i.e., $[\theta][I]_{c},(28)$ can be regarded as a solution to (24) with an over determined set of weighting functions. In a conventional moment method type of solution one would have to evaluate the elements of the matrix $[A]$ and then obtain its inverse. However, in the method described in this paper, we do not explicitly evaluate the elements of the matrix $[A]$, but the matrix product $[A][I]_{c}$ is rapidly computed with a minimum number of multiplications by the FFT algorithm. Also, since this product directly gives the near field, it provides a convenient means for checking the satisfaction of the boundary condition, a provision which would require extra computations in a conventional moment method solution.

The middle $\left(N_{p}+1\right)$ elements of the matrix [ $\left.\theta\right]$ are ones (corresponding to points defined on the antenna) and the rest are zeros. Therefore, from (29), matrix $[A]$ can be written in a partitioned form as $\left[[0]\left[A^{\prime \prime}\right][0]\right]$. where $\left[A^{\prime \prime}\right]$ is a $N \times$ $\left(N_{p}+1\right)$ matrix whose elements are the $-N_{p} / 2$ to $N_{p} / 2$ column elements of $\left[A^{\prime}\right]$ and the remaining elements of $[A]$ are zeros. It will be shown later that $N_{p}$ must be less than $N / 2$. In view of this, matrix $[A]$ becomes sparse and half or more than half of its elements can be zeros. Consequently equation (28) lends itself ideally to a solution by an iterative method.

To do this, (6) itself can be written in an iterative form as

$$
\begin{aligned}
& C\left[\frac{d^{2}}{d z^{2}}+\beta^{2}\right]\left(G * J^{k+1}\right) \\
& =-\theta E_{z i}+C \hat{\theta}\left[\frac{d^{2}}{d z^{2}}+\beta^{2}\right]\left(G * \theta J^{k}\right) .
\end{aligned}
$$

Fourier transforming this equation on both sides, the following equation is obtained

$$
\begin{aligned}
C[Q]\left[I^{k+1}\right]_{c}= & {[E]_{c}+C \Delta z[W][\hat{\theta}] \Delta f[\bar{W}] } \\
& \cdot[D G] \Delta z[W][\theta]\left[I^{k}\right]_{c}
\end{aligned}
$$

where $[Q]=\Delta z[D G][W]$ and the matrix $[\hat{\theta}]=[U]-[\theta]$. The diagonal matrix $[D G]$ is nonsingular $(11)$ and so is $[Q]$.

Solution to (28) can be written as

$$
\left[I^{k+1}\right]_{c}=\left[E_{a}\right]_{c}+[G]\left[I^{k}\right]_{c}
$$

where

$$
\left[E_{u}\right]_{c}=(1 / C)[Q]^{-1}[E]_{c}
$$

and

$$
\begin{aligned}
{[G] } & =\Delta f[\bar{W}][D G]^{-1} \Delta z[W][\hat{\theta}] \Delta f[\bar{W}][D G] \Delta z[W][\theta] \\
& =[\theta]-[Q]^{-1}[A]
\end{aligned}
$$

which is the iterative solution for the system given by (28).

The matrix $[G]$ is the $(N \times N)$ iteration matrix for this method. The method is of the first degree since $\left[I^{k+1}\right]_{c}$ depends explicitly on $\left[I^{k}\right]_{c}$ and not on $\left[I^{k-1}\right]_{c} \cdots\left[I^{0}\right]_{c}$. It is linear since neither $[G]$ nor $\left[E_{a}\right]_{c}$ depend on $\left[I^{k}\right]_{c}$ and is stationary since neither $[G]$ nor $\left[E_{a}\right]_{c}$ depend on $k$.

Convergence of the iteration process now requires the spectral radius of $[G]$ to be less than unity [13]. From (35) it can be seen that for a given $N$ and $N_{p}$, the only matrices whose elements depend on the antenna dimensions are $[D G]$ and $[D G]^{-1}$. Therefore the eigenvalues of the matrix $[G]$ would depend on the matrices $[D G]$ and $[D G]^{-1}$. This condition can be met by a proper choice of $N$ and $N_{p}$.

The advantages of truncating the current distribution (7) can now be explained. In the first place, since the Fourier inverse of the $k$ th transform of the current distribution does not give rise to a current that is zero outside the range of the antenna [9], this truncation can be viewed as an acceleration scheme itself since we use our a priori knowledge that the current distribution is zero outside the antenna. Secondly, this operation prevents 
circular convolution. While computing $F\left(G^{*} J\right)$, if the Green's function can be regarded as essentially support limited over the range of the antenna alone, and if $N_{p}<N / 2$, the operator $\theta$ does the same as that of adding the appropriate number of zeros to the current distribution, which is a suggested remedy for circular convolution [15]. Fourier transform of $\left(G^{*} J\right)$ then becomes the $N$ point DFT of two $N_{p}$ point sequences $G$ and $J$. Lastly, the truncation of the current distribution is essential for the iteration process. It can be seen from the elements of the matrix $[G]$ that in the absence of the matrix $[\theta]$ (which operates

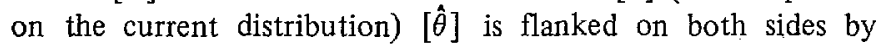
matrices which are the inverses of each other. Consequently the eigenvalues of the matrix $[G]$ will be the same as those of the matrix $[\hat{\theta}]$ which are one and zero. Hence the iteration process will not converge if the current distribution is not truncated before taking its transform.

\section{NUMERICAL RESULTS AND DISCUSSION}

Analysis of cylindrical antennas of various lengths has been carried out and the results are presented in this section. The method of solution being iterative, a good choice of the initial current distribution can significantly hasten the convergence. Since the sinusoidal distribution is known to be an excellent approximation for the current distribution on a cylindrical antenna, in all examples illustrated, the initial current distribution is assumed to be sinusoidally distributed in magnitude and of zero phase.

As mentioned earlier, the tangential electric field on the surface of the antenna can be taken as a measure of the convergence of the iteration process. However, since the occurrence of strong fields at the ends of the antenna is inevitable, we enforce the boundary conditions everywhere except the wire ends and define the error field as

$$
E_{\mathrm{rms}}=\left[\left(\sum_{n=-\left(N_{p} / 2\right)+1}^{\left(N_{p} / 2\right)-1}\left|E_{z i n}-F_{z n}\right|^{2}\right) /\left(N_{p}-1\right)\right]^{1 / 2}
$$

where $E_{z i n}$ and $F_{z n}$ denote the values of $E_{z i}$ and $F_{z}$ at points $n$.

The input impedance has been evaluated by using the stationary expression [8],

$$
Z_{\text {in }}=\frac{-\int_{-h}^{h} F_{z} \cdot J}{I_{\text {feed }}^{2}} .
$$

Note that this form depends not only on the electric field in the feed region but also that on the surface of the antenna. Moreover, it is also valid for widely distributed source fields. Since the computed value of the input impedance is a local function of the current at the feed region, for the sake of completeness, we have also considered a nonlocal parameter, namely the root mean squared current, defined as $[8]$,

$$
I_{\mathrm{rms}}=\left[\left(\sum_{n=-N_{p} / 2}^{N_{p} / 2}\left|I_{n}\right|^{2}\right) /\left(N_{p}+1\right)\right]^{1 / 2}
$$

Results pertaining to specific parameters are discussed below.

\section{A. Convergence of the Iteration Process}

Convergence of this method depends on the elements of the matrix $[D G]$ and its inverse, which govern the eigenvalues of the

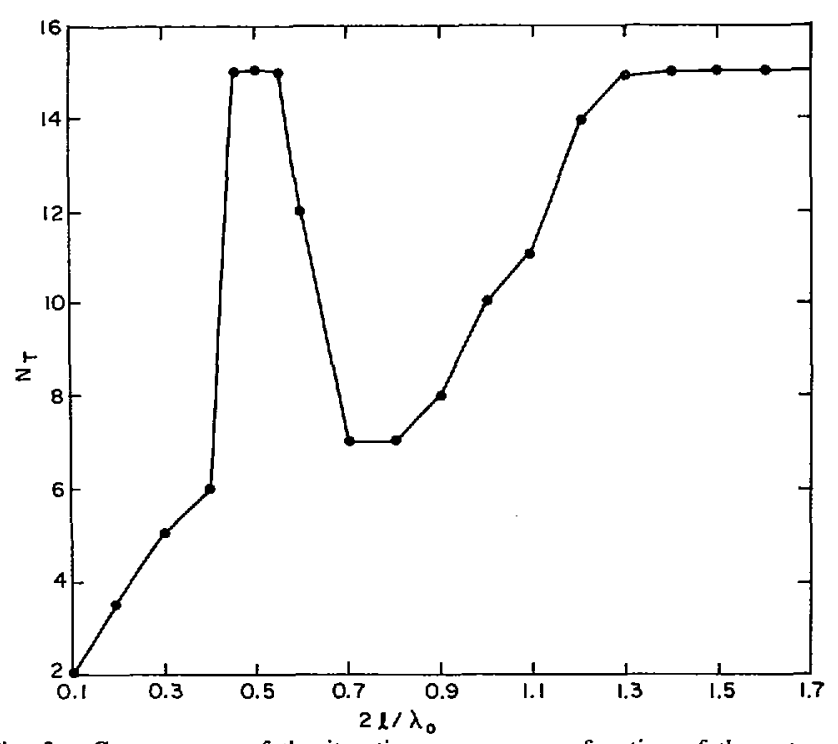

Fig. 2. Convergence of the iteration process as a function of the antenna length, $N=64, h / a=100, N_{p}=4,6,8, \cdots, 32$, and $N_{T}=$ number of values of $N_{p}$ for which the iterations converged.

matrix $[G]$. A study of the convergence for a wide range of antenna lengths, is illustrated in Fig. 2. These results were generated with the help of an acceleration scheme discussed in the next section. $N$ has been kept constant $(=64)$ and for every antenna length investigated, $N_{p}$ has been varied from four to 32 in steps of two. Of the 15 values, the number of values of $N_{p}$ for which the iteration process converged (denoted by $N_{T}$ ) is plotted as a function of the antenna length (Fig. 2). It can be seen that as the length increases, $N_{T}$ also increases, except in a small neighborhood of the half-wavelength where the iteration process is always convergent. The same trend has also been observed with a choice of $N=128$.

A possible explanation for this can be given by utilizing any consistent multiplicative matrix norm, the infinite matrix norm for instance, as an estimate of the spectral radius of $[G]$. From (11) it can be seen that, of the elements of the matrix $[D G]$, the element for which $m=n=-N / 2$ has the largest magnitude. The element of smallest magnitude is in the neighborhood of $m=$ $n=0$ and is found to be nearly the same for antennas of various lengths. It is easy to see that the infinite matrix norm of $[G]$ can be kept at a minimum by keeping $\Delta f$ small. However, since the infinite matrix norm only gives an upper estimate of the spectral radius of $[G]$, this method is also found to converge for values of $N_{p}$ which result in slightly larger values of $\Delta f$. This is to be expected since the eigenvalues of $[G]$ would be complicated functions of the elements of $[D G]$. It must be mentioned that for every antenna length we have investigated, there is always at least one $N_{p}$ for which the iteration process converges and sufficiently large enough so as to result in a reasonably good input impedance.

\section{B. An Acceleration Procedure}

It is observed that often the main difference between the approximate $J^{k}$ derived after a few iterations and the exact solution is a complex factor. We have therefore investigated the use of a variational weight factor defined as [11],

$$
X^{k}=\frac{\left\langle\theta J^{k},-E_{z i}\right\rangle}{\left\langle\theta J^{k}, F_{z}\right\rangle}
$$




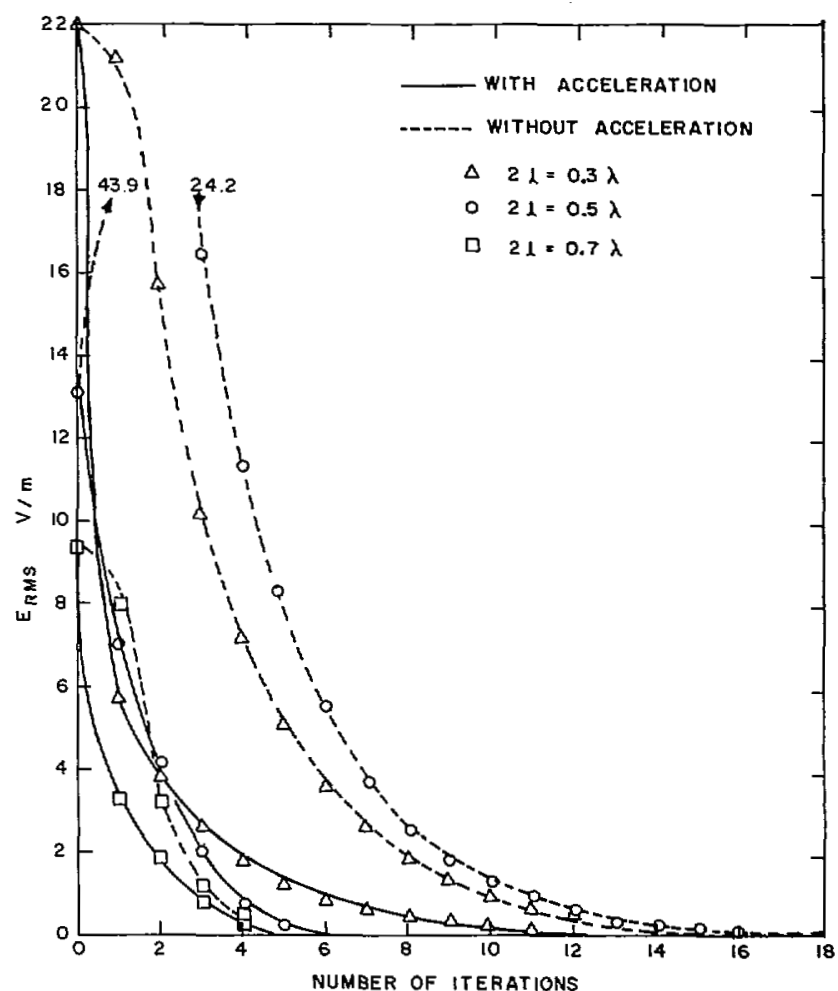

Fig. 3. Convergence with and without acceleration, $N=256, N_{p}=42, h / a$ $=100$, delta gap feed.

where $\langle\cdot\rangle$ denotes the inner product and $F_{z}$ is a tangential electric field due to $J^{k}$. The current distribution $J^{k}$ is multiplied by $X^{k}$ after each iteration. With the use of this factor, one can start the iteration with a sinusoidal current distribution $J^{0}$ of an arbitrary amplitude. At the end of the first iteration, the current distribution $J^{1}$ multiplied by the factor $X^{1}$ will result in a near field comparable to $-E_{z i}$. It is found that convergence is significantly accelerated by this method and is illustrated in Fig. 3. This is also helpful in situations where the iteration process is very slowly convergent or begins to oscillate.

The above procedure may alternatively be viewed as a multiplication of the iteration matrix $[G]$ itself by a complex constant. Note that the rate of convergence will be higher the smaller the spectral radius $[G]$. Since the constant $X^{k}$ tends to minimize the deviation between $-E_{z i}$ and $F_{z}$, it is reasonable to presume that this factor after multiplying the matrix $[G]$ will reduce the spectral radius of $[G]$ and hence accelerate the convergence. The factor $X^{k}$ will gradually tend to unity as the number of iterations increase and consequently the rate of convergence reduces with increasing number of iterations. It can also be seen from Fig. 3 that convergence with acceleration is monotonic and the residual $E_{\mathrm{rms}}$ does not oscillate. Although this acceleration procedure has no adverse effect on the unaccelerated iteration, it does not guarantee convergence always. An example of divergence is illustrated in Fig. 4. Referring to Figs. 3 and 4, it can be seen that for the $0.3 \lambda$ case, for the same $N_{p}(=42)$, the iteration converged for $N=256$ and diverged for $N=128$. Whereas, for the $0.7 \lambda$ case, for the same $N(=256)$, the iteration converged for $N_{p}=42$ but diverged for $N_{p}=44$. Thus, $N_{p}$ and $N$ play an important role in this method. Note that the oscillatory behavior in Fig. 4 is on account of acceleration without this $E_{\mathrm{rms}}$ builds up almost exponentially.

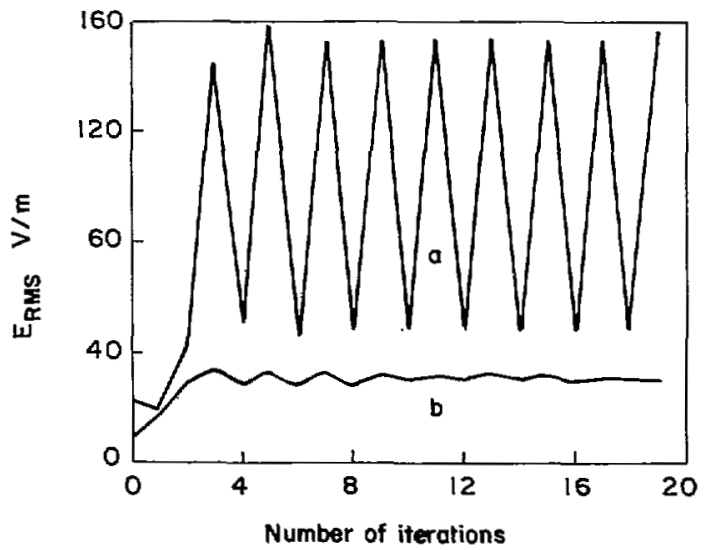

Fig. 4. Illustration of divergence (a) $2 h=0.3, \lambda, N=128, N_{p}=42$. (b) $2 h$ $=0.7 \lambda, N=256, N_{p}=44$.

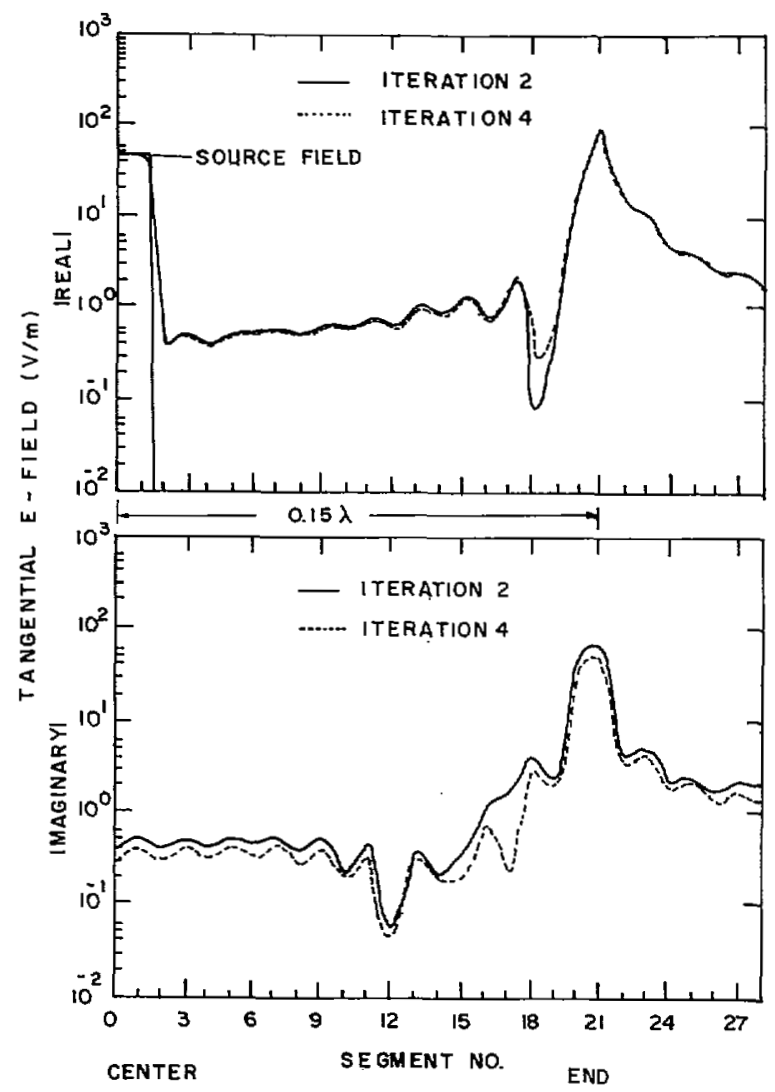

Fig. 5. Near fields of a $0.3 \lambda$ dipole with a three-segment pulse feed. $h / a=$ $100, N=256, N_{p}=42$.

\section{Near Fields}

The computed tangential electric fields for three different types of source distributions are shown in Figs. 5-7. It can be seen from Figs. 5 and 6 that in situations where the source field can be represented exactly in terms of pulse basis functions (like for the delta gap and the pulse feed) the boundary conditions at the feed region are met exactly right after the second iteration itself. Further iterations improve the near field away from the feed region. This is to be expected since the FFT itself employs pulse functions as its basis. However, for continuous field distributions such as the magnetic frill (Fig. 7), a larger 


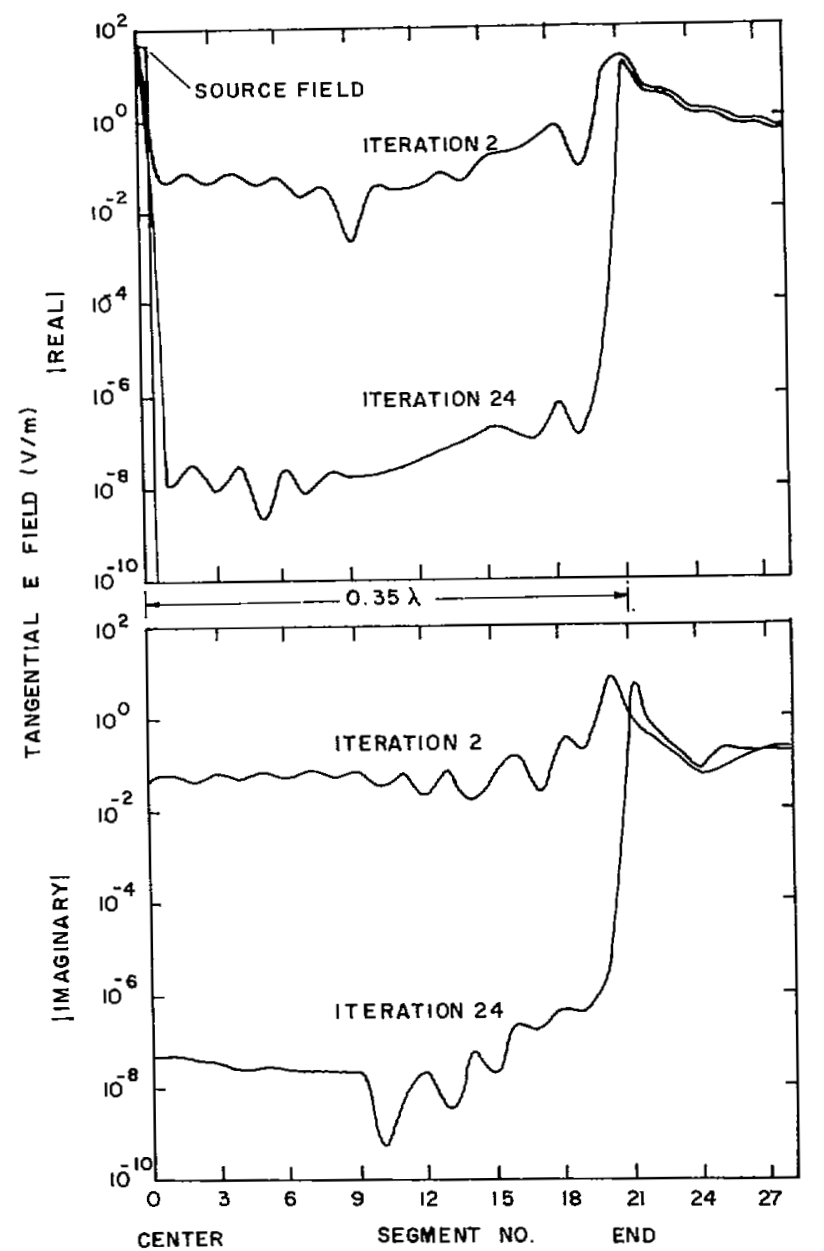

Fig. 6. Near fields of a $0.7 \lambda$ dipole with a delta gap feed $h / a=100, N=$ $256, N_{p}=42$.

number of iterations will be required to achieve an adequate representation of the near field. Also a larger $N_{p}$ is required to satisfy the boundary conditions adequately.

\section{Input Impedance}

The input impedance has been calculated using (37). Results are illustrated for three cases in Fig. 8. For the $0.3 \lambda$ and $0.7 \lambda$ cases our results compare well with those of Mittra and Klein [8] with a slightly larger $N_{p}$, i.e., with $N_{p}=30$ we obtain the same impedances as those obtained in [8] and 19 piecewise sinusoidal basis functions. However, as $N_{p}$ is increased, the input resistance for the $0.7 \lambda$ case does not increase in the manner as illustrated in [8]. For example, with $N_{p}=84$ (not shown here) the input resistance for the $0.7 \lambda$ case is $494 \Omega$ in contrast with $\cong 570 \Omega$ (with $\cong 60$ piecewise sinusoids [8]). This is to be expected since when the iteration process converges the boundary conditions, except at the wire ends, are almost exactly met, and the current distribution is zero at the wire ends. Consequently it is only the current distribution at the feed point that governs the input impedance. Since the rms current (Fig. 9) also converges it is unlikely for the input impedance to vary largely as the number of basis functions are increased. Also there will be no difference between using the stationary expression for $Z_{\text {in }}$ (37) and the usual equation $Z_{\text {in }}=V / I_{\text {feed }}$. Our results also compare well with experimental results of input

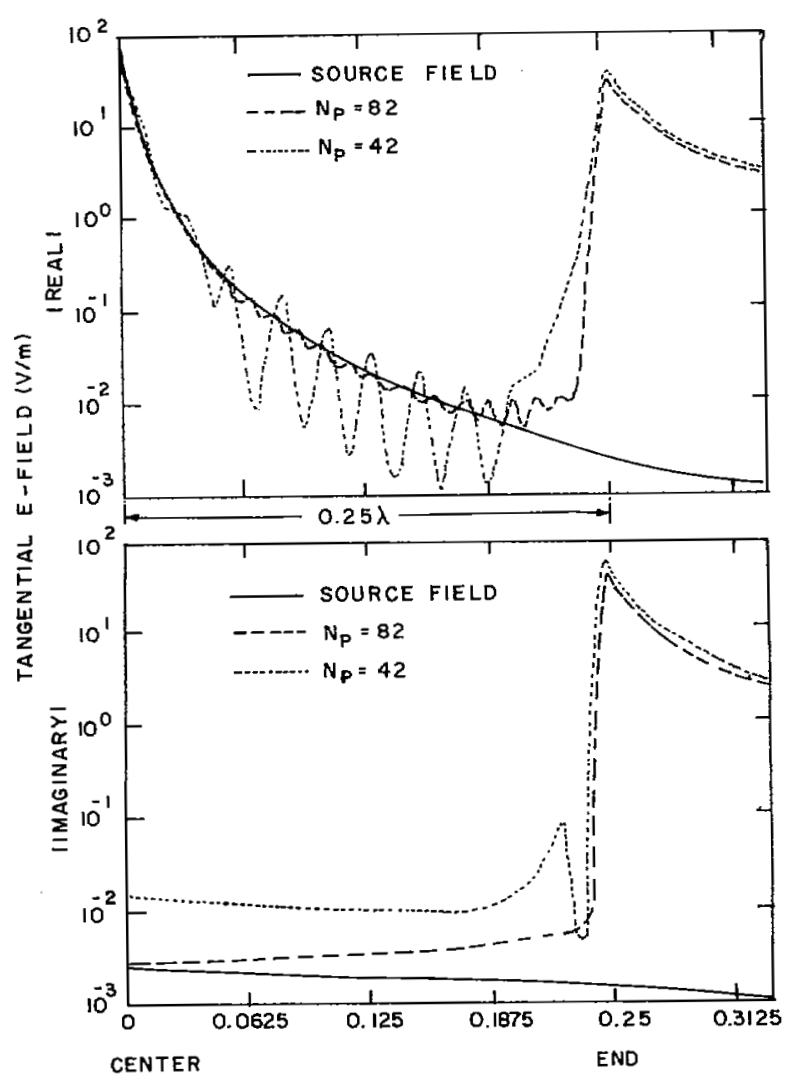

Fig. 7. Near fields of $0.5 \lambda$ dipole with a magnetic frill excitation. $h / a=$ $100, b / a=1.2, N=256$.

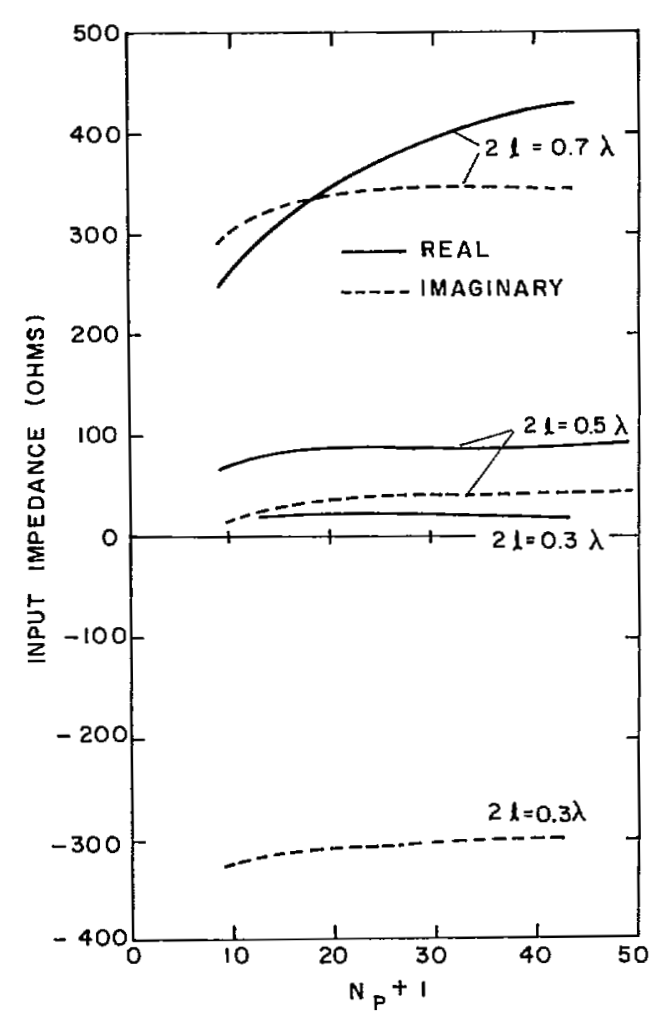

Fig. 8. Convergence of the input impedance. $h / a=100$, delta gap feed. 


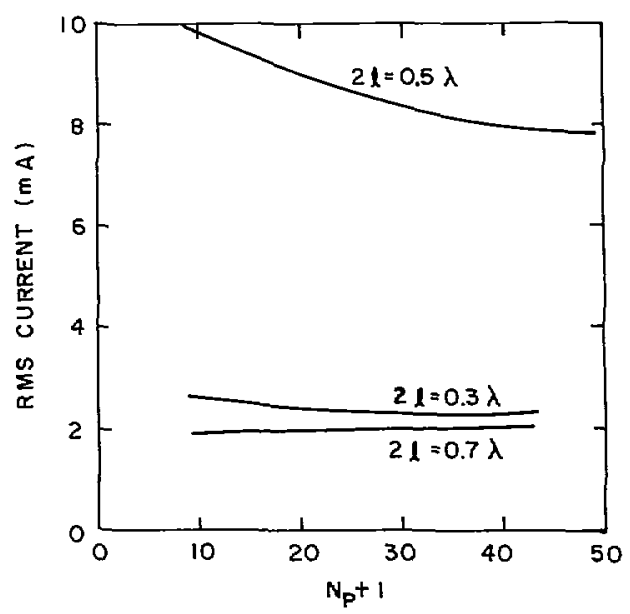

Fig. 9. Convergence of the rms current. $h / a=100$, delta gap feed.

impedance as measured by Mack [1]. Convergence of the rms current is also in a good agreement with the results of Mittra and Klein [8].

\section{CONCLUSION}

It has been shown that the spectral iteration technique provides a numerically efficient and accurate procedure for solving cylindrical antenna problems. Advantages of this method compared to conventional moment method solutions are clearly brought out and convergence criteria have been established. Application of a variational weighting factor for accelerating the convergence has been illustrated. It must be pointed out that of the many acceleration schemes tried out by the authors including the steepest descent acceleration, the above scheme proved to be most promising. Numerical comparisons of the input impedance have been made with results deduced elsewhere and are found to be in good agreement. Since the satisfaction of the boundary conditions is always ensured in this method, the near fields obtained will be more accurate than those obtained by other methods. This technique can also be extended to the analysis of arrays of cylindrical antennas. The fact that this method is always convergent for dipole lengths in the neighborhood of the half-wavelength indicates that this method could be effectively used in the analysis of large arrays of resonant length dipoles.

\section{REFERENCES}

[1] R. Mittra, Ed., Computer Techniques for Electromagnetics. Oxford: Pergamon, 1973.

[2] R. W. P. King, S. S. Sandler, and R. B. Mack, Arrays of Cylindrical Dipoles. Cambridge: Cambridge Univ., 1968.

[3] R. F. Harrington, Field Computation by Moment Methods. New York: Macmillan, 1968.

[4] E. K. Miller and F. J. Deadrick, "Some computational aspects of thin wire modelling," in Numerical and Asymptotic Techniques in Electromagnetics, R. Mittra, Ed. New York: Springer-Verlag, 1975.
[5] R. W. P. King and T. T. Wu, "The electrical field very near a cylindrical driven antenna," Radio Sci., vol. I. pp. 353-359, Mar. 1966.

[6] H. P. Neff, Jr., C. A. Ciller, Jr., and J. D. Tillman, Jr., "A simple approximation to the current distribution on a thin centerfed linear antenna," IEEE Trans. Antennas Propagat., vol. AP-18, pp. 399400, May 1970.

[7] R. Anders, "Minimum continuity requirements for basis functions used with the Pocklington's integral equation," in Digest URSI Symp. Electromagne. Wave Theory, 1977, pp. 284-287.

[8] R. Mittra and C. A. Klein, "Stability and convergence of moment method solutions," in Numerical and Asymptotic Technqiues in Electromagnetics, R. Mittra, Ed. New York: Springer-Verlag, 1975.

[9] W. L. Ko and R. Mittra, "A new approach based on a combination of integral equation and asymptotic techniques for solving electromagnetic scattering problems," IEEE Trans. Antennas Propagat., vol. AP-25, pp. 187-197, Mar. 1977.

[10] C. H. Tsao and R. Mittra, "A spectral iteration technique for analyzing scattering from frequency selective surfaces," IEEE Trans. Antennas Propagat., vol. AP-30, pp. 303-308, Mar. 1982.

[11] R. Kastner and R. Mittra, "A spectral iteration technique for analyzing a corrugated surface twist polarizer for scanning reflector antennas," IEEE Trans. Antennas Propagat., vol. AP-30, pp. 673-676, July 1982.

[12] _- "A spectral iteration technique for analyzing scattering from arbitrary bodies, Part I: Cylindrical scatterers with $E$-wave incidence," IEEE Trans. Antennas Propagat., vol. AP-31, pp. 499-506, May 1983.

[13]T. K. Sarkar, K. R. Siarkiewicz, and R. F. Stratton, "Survey of numerical methods for the solution of large systems of linear equations for electromagnetic field problems," IEEE Trans. Antennas Propagat., vol. AP-29, pp. 847-856, 1981.

[14] J. W. Cooley, P. Lewis, and P. D. Welch, "The fast Fourier transform algorithm: Programming considerations in the calculation of sine, cosine and Laplace transforms," J. Sound Vib., vol. 12, pp. 315-337, 1970.

[15] L. R. Rabiner and B. Gold, Theory and Application of Digital Signal Processing. Englewood Cliffs, NJ: Prentice Hall, 1975.

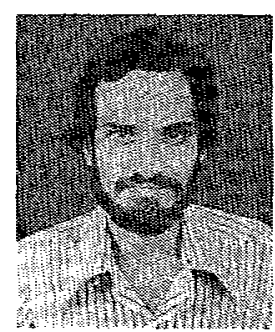

S. A. Bokhari was born in Bangalore, India. He received the B.E. degree in electronics engineering from the Visveswaraya College of Engineering, Bangalore, in 1980, and the M.Sc.(Eng) degree from the Department of Electrical Communication Engineering, Indian Institute of Science, Bangalore, in 1983 .

He is currently working toward a Ph.D. degree in the Department of Aerospace Engineering, Indian Institute of Science, Bangalore. His areas of research interest are antenna array theory, numerical methods and signal processing.

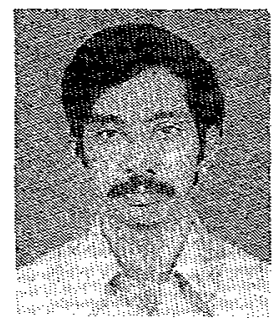

N. Balakrishnan received the B.E. degree in electronics and communication from the University of Madras, India in 1972, and the Ph.D. degree in antennas from the Indian Institute of Science, Bangalore, India, in 1979.

In 1973 he joined the Department of Aeronautical Engineering, Indian Institute of Science, where he is currently employed as an Assistant Professor. His fields of interest are numerical electromagnetics, computer science, and missile guidance. 